\title{
Up-down coefficients for permutations
}

Vladimir Shevelev and Juergen Spilker

\begin{abstract}
Vladimir Shevelev wurde 1945 geboren in Novocherkassk (UdSSR). Er ist Professor an der Universität in Beer-Sheva (Israel). Seine speziellen Interessen sind Zahlentheorie und Kombinatorik.

Jürgen Spilker wurde 1935 in Berlin geboren. Er ist Professor im Ruhestand an der Universität in Freiburg (Breisgau). Seine Interessen sind Zahlentheorie und Analysis.
\end{abstract}

\section{Definitions and introduction}

Let $\mathbf{S}_{n}$ be the set of permutations of the numbers $1,2, \ldots, n$. How many $\pi=\left(\pi_{1}, \pi_{2}, \ldots\right.$, $\left.\pi_{n}\right) \in \mathbf{S}_{n}$ are alternating, that is $\pi_{1}<\pi_{2}>\pi_{3}<\cdots$ ? This question was answered by André in 1881 [2]. He proved that the exponential generating function of numbers of such permutations is the sum of tangent and secant (Section 6).

In 1968, Niven generalized this problem [11]. For every $\pi=\left(\pi_{1}, \pi_{2}, \ldots, \pi_{n}\right) \in \mathbf{S}_{n}$ he defines the signature $Q=\left(q_{1}, q_{2}, \ldots, q_{n-1}\right)$ by

$$
q_{i}:=\left\{\begin{aligned}
1, & \text { if } \pi_{i}<\pi_{i+1} \\
-1, & \text { if } \pi_{i}>\pi_{i+1}
\end{aligned}\right.
$$

and proves by a determinant a formula of the number $[Q]$ of all $\pi \in \mathbf{S}_{n}$ with given signature $Q$.

An jeder Stelle $i$ einer Permutation $\pi=\left(\pi_{1}, \pi_{2}, \ldots, \pi_{n}\right)$ der Zahlen $1,2, \ldots, n$ gilt entweder $\pi_{i}<\pi_{i+1}$ ("up") oder $\pi_{i}>\pi_{i+1}$ ("down"). Dabei geben etwa die Eulerschen Zickzackzahlen die Anzahl alternierender Permutationen an, bei denen sich "up" und "down" abwechseln und dabei mit "up" beginnen. Diese Zahlen tauchen in der Potenzreihenentwicklung der Tangens- und der Sekansfunktion auf, wie Désiré André 1881 feststellte. Notiert man die "ups" und "downs" geeignet als Dualzahl, so erhält man den Index $k$ der Permutation $\pi$. Für die Anzahl der Permutationen mit gegebenem Index $k$ haben die Autoren das Symbol $\left\{\begin{array}{l}n \\ k\end{array}\right\}$ eingeführt. Es ähnelt dem Binomialkoeffizienten $\left(\begin{array}{l}n \\ k\end{array}\right)$ und genügt unerwarteten neuen Gleichungen und Ungleichungen. 
Since this Niven's celebrated result, many mathematicians obtained important results in this topic (for example, see papers [3]-[6], [8], [10], [12], [14]-[16]). However, in spite of many works on this topic, the technique of enumeration and estimation of the number of permutation with a fixed Niven signature remained rather weak and was based mainly on Niven's determinant only. An exception to the general rule worth mentioning is André's alternating permutations. Niven wrote (see Section 4, p. 119 in [11]) that he was unable to formulate general equalities and inequalities for the numbers of permutations having different signatures. By this, he posed an important problem to get such relations. Recently in [13], the first-named author introduced a new symbol $\left\{\begin{array}{l}n \\ k\end{array}\right\}$ and found methods to compute it.

Definition. Let $\pi \in \mathbf{S}_{n}$ with signature $Q=\left(q_{1}, q_{2}, \ldots, q_{n-1}\right)$.

a) We call the dual number

$$
k=k(Q)=\sum_{1 \leq i \leq n-1:} 2_{q_{i}=1}^{n-1-i}
$$

the index of $\pi$.

b) We denote by $\left\{\begin{array}{l}n \\ k\end{array}\right\}$ the number of permutations of $\mathbf{S}_{n}$ with index $k$ and call it the up-down coefficient of $\pi$.

For every natural $n$, the map from the signatures to the indices

$$
\{1,-1\}^{n-1} \rightarrow\left\{0,1, \ldots, 2^{n-1}-1\right\}: Q \mapsto k(Q)
$$

is bijective. So, the new symbol $\left\{\begin{array}{l}n \\ k\end{array}\right\}$ equals the number $[Q]$ of permutations of $\mathbf{S}_{n}$ with Niven's signature $Q$.

Example. The permutation $\pi=(2,1,4,3) \in \mathbf{S}_{4}$ has signature $Q=(-1,1,-1)$ and index 2. The other permutations of index 2 are $(3,1,4,2),(3,2,4,1),(4,1,3,2)$ and $(4,2,3,1)$; so $\left\{\begin{array}{l}4 \\ 2\end{array}\right\}=5$.

In [13] it is shown that $\left\{\begin{array}{l}n \\ k\end{array}\right\}$ is a polynomial in $n$ with rational coefficients for every fixed $k$, and also a recursive method to compute these coefficients and a table of the $\left\{\begin{array}{l}n \\ k\end{array}\right\}, 0 \leq k \leq$ 31 , are given.

In this note we reformulate results of Niven [11] in the context of up-down coefficients, prove new equalities (Section 3) and inequalities (Section 4) between symbols and describe their connection with binomial coefficients (Section 2) and Euler and Bernoulli numbers (Section 6). It is astonishing that up-dow coefficients contain such a large algebraic structure.

\section{Up-down coefficients and binomial coefficients}

The following table contains simple properties of up-down coefficients $\left\{\begin{array}{l}n \\ k\end{array}\right\}$ and binomial coefficients $\left(\begin{array}{l}n \\ k\end{array}\right)$. Below $n$ is a natural number. 
(a) $1 \leq\left\{\begin{array}{l}n \\ k\end{array}\right\} \leq n$ ! $\left(0 \leq k<2^{n-1}\right), \quad 1 \leq\left(\begin{array}{l}n \\ k\end{array}\right) \leq 2^{n} \quad(0 \leq k \leq n)$

(b) $\left\{\begin{array}{l}n \\ k\end{array}\right\}=\left\{\begin{array}{c}n \\ 2^{n-1}-1-k\end{array}\right\}, \quad\left(\begin{array}{l}n \\ k\end{array}\right)=\left(\begin{array}{c}n \\ n-k\end{array}\right)$

(c) $\sum_{0 \leq k<2^{n-1}}\left\{\begin{array}{l}n \\ k\end{array}\right\}=n !, \quad \sum_{0 \leq k \leq n}\left(\begin{array}{l}n \\ k\end{array}\right)=2^{n}$

(d) $\sum_{0 \leq k<2^{n-1}}(-1)^{k}\left\{\begin{array}{l}n \\ k\end{array}\right\}=0(n>1), \quad \sum_{0 \leq k \leq n}(-1)^{k}\left(\begin{array}{l}n \\ k\end{array}\right)=0$

(e) $\sum_{\substack{0 \leq k<2^{n-1} \\ k \text { even }}}\left\{\begin{array}{l}n \\ k\end{array}\right\}=\sum_{\substack{0 \leq k<2^{n-1}, k \text { odd }}}\left\{\begin{array}{l}n \\ k\end{array}\right\}=\frac{n !}{2}(n>1)$,

$$
\sum_{\substack{0 \leq k \leq n \\
k \text { even }}}\left(\begin{array}{l}
n \\
k
\end{array}\right)=\sum_{\substack{0 \leq k \leq n \\
k \text { odd }}}\left(\begin{array}{l}
n \\
k
\end{array}\right)=2^{n-1}
$$

(f) $\left\{\begin{array}{c}n \\ 2^{k-1}-1\end{array}\right\}+\left\{\begin{array}{c}n \\ 2^{k}-1\end{array}\right\}=\left\{\begin{array}{c}n+1 \\ 2^{k}-1\end{array}\right\}, \quad\left(\begin{array}{c}n \\ k-1\end{array}\right)+\left(\begin{array}{l}n \\ k\end{array}\right)=\left(\begin{array}{c}n+1 \\ k\end{array}\right)$.

Proof of (a)-(f). (a) The upper estimate is trivial, since the cardinality of $\mathbf{S}_{n}$ is $n$ ! We prove that $\left\{\begin{array}{l}n \\ k\end{array}\right\} \neq 0$ by induction on $n$. Suppose that the statement is valid for $n-1,0 \leq k<2^{n-2}$. Now let $0 \leq k<2^{n-1}$. Distinguish two cases.

1) $k=2 l+1$. Since $0 \leq l<2^{n-2}$, there exists $\left(\pi_{1}, \pi_{2}, \ldots, \pi_{n-1}\right) \in \mathbf{S}_{n-1}$ with index $l$. Then $\left(\pi_{1}, \pi_{2}, \ldots, \pi_{n-1}, n\right)$ has index $2 l+1$. Hence $\left\{\begin{array}{l}n \\ k\end{array}\right\} \geq 1$.

2) $k=2 l$. If $\left(\pi_{1}, \pi_{2}, \ldots, \pi_{n-1}\right) \in \mathbf{S}_{n-1}$ has index $l$, then $\left(\pi_{1}+1, \pi_{2}+1, \ldots, \pi_{n-1}+\right.$ $1,1) \in \mathbf{S}_{n}$ has index $2 l$. Hence $\left\{\begin{array}{l}n \\ k\end{array}\right\} \geq 1$.

Since, evidently, $\left\{\begin{array}{l}n \\ 0\end{array}\right\}=1$, the proof is complete.

(b) The map $F:\left(\pi_{1}, \pi_{2}, \ldots, \pi_{n}\right) \mapsto\left(n+1-\pi_{1}, n+1-\pi_{2}, \ldots, n+1-\pi_{n}\right)$ is a bijection of $\mathbf{S}_{n}$. If $\pi$ has index $k=\sum_{1 \leq i<n: q_{i}=1} 2^{n-1-i}$, then $F(\pi)$ has index

$$
\sum_{1 \leq i<n: q_{i}=-1} 2^{n-1-i}=\sum_{1 \leq i<n} 2^{n-1-i}-\sum_{1 \leq i<n: q_{i}=1} 2^{n-1-i}=2^{n-1}-1-k .
$$

(c) Both sides count all $n$ ! permutations of $\mathbf{S}_{n}$.

(d) $\sum_{0 \leq k<2^{n-1}}(-1)^{k}\left\{\begin{array}{l}n \\ k\end{array}\right\}=\sum_{0 \leq k<2^{n-2}}(-1)^{k}\left(\left\{\begin{array}{l}n \\ k\end{array}\right\}-\left\{\begin{array}{c}n \\ 2^{n-1}-1-k\end{array}\right\}\right)=0$

according to (b).

(e) Add or subtract (c) and (d).

(f) The number of permutations of $\mathbf{S}_{n+1}$ with index $2^{k-1}+2^{k-2}+\cdots+1$ is the same as the number of permutations of $\mathbf{S}_{n}$ with index $l 2^{k-1}+2^{k-2}+\cdots+1$ with $l=0$ or 1 . 
Note that, by property (f), we have the following Pascal-like triangle

$$
\begin{aligned}
& \left\{\begin{array}{l}
1 \\
0
\end{array}\right\} \\
& \left\{\begin{array}{l}
2 \\
0
\end{array}\right\} \quad\left\{\begin{array}{l}
2 \\
1
\end{array}\right\} \\
& \left\{\begin{array}{l}
3 \\
0
\end{array}\right\} \quad\left\{\begin{array}{l}
3 \\
1
\end{array}\right\} \quad\left\{\begin{array}{l}
3 \\
3
\end{array}\right\} \\
& \left\{\begin{array}{l}
4 \\
0
\end{array}\right\} \quad\left\{\begin{array}{l}
4 \\
1
\end{array}\right\} \quad\left\{\begin{array}{l}
4 \\
3
\end{array}\right\} \quad\left\{\begin{array}{l}
4 \\
7
\end{array}\right\} \\
& \begin{cases}\left\{\begin{array}{l}
n \\
0
\end{array}\right\} \cdots\left\{2_{2^{\frac{n-3}{2}}-1}^{n}\right\}\left\{\begin{array}{c}
n \\
2^{\frac{n-1}{2}}-1
\end{array}\right\} \cdots\left\{\begin{array}{c}
n \\
2^{n-1}-1
\end{array}\right\}, & \text { if } n \text { is odd, } \\
\left.\left\{\begin{array}{l}
n \\
0
\end{array}\right\} \cdots 2_{2^{\frac{n-2}{2}}-1}^{n}\right\}\left\{2_{2^{\frac{n}{2}}-1}^{n}\right\} \cdots\left\{\begin{array}{c}
n \\
2^{n-1}-1
\end{array}\right\}, & \text { if } n \text { is even. }\end{cases}
\end{aligned}
$$

with the $n$th line

\section{Equalities for up-down coefficients}

Theorem 1. If $n \geq 1,0 \leq k<2^{n-1}, m \geq 1,0 \leq l<2^{m-1}$, then the following identities hold

$$
\begin{gathered}
\left\{\begin{array}{c}
n+m \\
2^{m} k+l
\end{array}\right\}+\left\{\begin{array}{c}
n+m \\
2^{m} k+l+2^{m-1}
\end{array}\right\}=\left(\begin{array}{c}
n+m \\
m
\end{array}\right)\left\{\begin{array}{c}
m \\
l
\end{array}\right\}\left\{\begin{array}{l}
n \\
k
\end{array}\right\}, \\
\left\{\begin{array}{c}
n+m \\
\left(2^{n-1}-1\right) 2^{m}+l-2^{m} k
\end{array}\right\}+\left\{\begin{array}{c}
n+m \\
\left(2^{n}-1\right) 2^{m-1}+l-2^{m} k
\end{array}\right\}=\left(\begin{array}{c}
n+m \\
m
\end{array}\right)\left\{\begin{array}{c}
m \\
l
\end{array}\right\}\left\{\begin{array}{l}
n \\
k
\end{array}\right\} .
\end{gathered}
$$

Here $\left(\begin{array}{c}n+m \\ m\end{array}\right)$ denotes the binomial coefficient.

Proof. By (a), there exist $Q \in\{ \pm 1\}^{n-1}$ with index $k$ and $P \in\{ \pm 1\}^{m-1}$ with index $l$. Further, we use Niven's theorem from [11]:

$$
[Q,-1, P]+[Q, 1, P]=\left(\begin{array}{c}
n+m \\
m
\end{array}\right)[P][Q] .
$$

Rewriting this for indices gives (3.1).

The second equality follows from

$$
[-Q,-1, P]+[-Q, 1, P]=\left(\begin{array}{c}
n+m \\
m
\end{array}\right)[P][-Q]=\left(\begin{array}{c}
n+m \\
m
\end{array}\right)[P][Q],
$$

since $-Q$ has index $2^{n-1}-1-k$; see (b). 
Remark 1. For a fixed $k$, one can formally consider the map $n \longmapsto\left\{\begin{array}{l}n \\ k\end{array}\right\}$ as a polynomial in $n$ with integer (positive and non-positive) values. So, (3.1) and (3.2) are valid for all $n, m \in \mathbb{N}, \quad k, l \in \mathbb{N}_{0}$.

Corollary 1. The following identities hold:

$$
\begin{aligned}
\left\{\begin{array}{c}
n+1 \\
2 k
\end{array}\right\}+\left\{\begin{array}{c}
n+1 \\
2 k+1
\end{array}\right\} & =(n+1)\left\{\begin{array}{l}
n \\
k
\end{array}\right\} ; \\
\left\{\begin{array}{c}
n+2 \\
4 k
\end{array}\right\}+\left\{\begin{array}{c}
n+2 \\
4 k+2
\end{array}\right\} & =\left\{\begin{array}{c}
n+2 \\
4 k+1
\end{array}\right\}+\left\{\begin{array}{c}
n+2 \\
4 k+3
\end{array}\right\}=\left(\begin{array}{c}
n+2 \\
2
\end{array}\right)\left\{\begin{array}{l}
n \\
k
\end{array}\right\} ; \\
\left\{\begin{array}{c}
n+3 \\
8 k
\end{array}\right\}+\left\{\begin{array}{c}
n+3 \\
8 k+4
\end{array}\right\} & =\left\{\begin{array}{c}
n+3 \\
8 k+3
\end{array}\right\}+\left\{\begin{array}{c}
n+3 \\
8 k+7
\end{array}\right\}=\left(\begin{array}{c}
n+3 \\
3
\end{array}\right)\left\{\begin{array}{l}
n \\
k
\end{array}\right\} ; \\
\left\{\begin{array}{c}
n+3 \\
8 k+1
\end{array}\right\}+\left\{\begin{array}{c}
n+3 \\
8 k+5
\end{array}\right\} & =\left\{\begin{array}{c}
n+3 \\
8 k+2
\end{array}\right\}+\left\{\begin{array}{c}
n+3 \\
8 k+6
\end{array}\right\}=2\left(\begin{array}{c}
n+3 \\
3
\end{array}\right)\left\{\begin{array}{l}
n \\
k
\end{array}\right\} ; \\
\left\{\begin{array}{l}
n \\
l
\end{array}\right\}+\left\{\begin{array}{c}
n \\
l+2^{m-1}
\end{array}\right\} & =\left(\begin{array}{c}
n \\
m
\end{array}\right)\left\{\begin{array}{l}
m \\
l
\end{array}\right\}, \text { if } n>m .
\end{aligned}
$$

Evidently, since the number of $\pi \in \mathbf{S}_{n}$ with the signature $\left(q_{1}, q_{2}, \ldots, q_{n-1}\right)$ equals the number of $\pi \in \mathbf{S}_{n}$ with the signature $\left(q_{n-1}, q_{n-2}, \ldots, q_{1}\right)$, we have

$$
\left\{\begin{array}{c}
n \\
\sum_{i=0}^{n-2} b_{i} 2^{n-i-2}
\end{array}\right\}=\left\{\begin{array}{c}
n \\
\sum_{i=0}^{n-2} b_{i} 2^{i}
\end{array}\right\}
$$

where $b_{i}$ are 0 or 1 .

In particular, we have identities

such that

$$
\begin{aligned}
& \left\{\begin{array}{c}
n \\
2^{n-3}
\end{array}\right\}=\left\{\begin{array}{l}
n \\
2
\end{array}\right\}, \quad\left\{\begin{array}{c}
n \\
2^{n-2}+2^{n-3}
\end{array}\right\}=\left\{\begin{array}{l}
n \\
3
\end{array}\right\}, \\
& \left\{\begin{array}{c}
n \\
2^{n-4}
\end{array}\right\}=\left\{\begin{array}{l}
n \\
4
\end{array}\right\}, \quad\left\{\begin{array}{c}
n \\
2^{n-2}+2^{n-4}
\end{array}\right\}=\left\{\begin{array}{l}
n \\
5
\end{array}\right\}, \ldots
\end{aligned}
$$

$$
\left\{\begin{array}{c}
n \\
2^{n-t}
\end{array}\right\}=\left\{\begin{array}{c}
n \\
2^{t-2}
\end{array}\right\}, \quad\left\{\begin{array}{c}
n \\
2^{n-2}+2^{n-t}
\end{array}\right\}=\left\{\begin{array}{c}
n \\
2^{t-2}+1
\end{array}\right\}
$$

and also

$$
\left\{\begin{array}{c}
n \\
2^{n-1}-2^{n-t}
\end{array}\right\}=\left\{\begin{array}{c}
n \\
2^{t-1}-1
\end{array}\right\} \text {, etc. }
$$

Besides, using (3.7), for $1 \leq r \leq t \leq n-2$, we have

$$
\left\{\begin{array}{c}
n \\
2^{t-r}+2^{t}
\end{array}\right\}=\left(\begin{array}{c}
n \\
t+1
\end{array}\right)\left\{\begin{array}{c}
t+1 \\
2^{t-r}
\end{array}\right\}-\left\{\begin{array}{c}
n \\
2^{t-r}
\end{array}\right\} .
$$

In particular, if $t=r$, then we find

$$
\left\{\begin{array}{c}
n \\
2^{r}+1
\end{array}\right\}=\left(\begin{array}{c}
n \\
r+1
\end{array}\right)\left\{\begin{array}{c}
r+1 \\
1
\end{array}\right\}-\left\{\begin{array}{c}
n \\
1
\end{array}\right\}=r\left(\begin{array}{c}
n \\
r+1
\end{array}\right)-n+1
$$


In addition, we give another explanation of the triangle in Section 2. For this, we prove the identity

$$
\left(\begin{array}{l}
n \\
r
\end{array}\right)=\left\{\begin{array}{c}
n+1 \\
2^{r}-1
\end{array}\right\}
$$

Indeed, using induction over $r$, let us find $\left\{\begin{array}{c}n+1 \\ 2^{r+1}-1\end{array}\right\}$. Take in (3.7) $l=2^{r}-1, \quad m=r+1$. Then we have

$$
\left\{\begin{array}{c}
n+1 \\
2^{r+1}-1
\end{array}\right\}=\left(\begin{array}{c}
n+1 \\
r+1
\end{array}\right)\left\{\begin{array}{c}
r+1 \\
2^{r}-1
\end{array}\right\}-\left\{\begin{array}{c}
n+1 \\
2^{r}-1
\end{array}\right\} \text {. }
$$

Note that $\left\{\begin{array}{c}r+1 \\ 2^{r}-1\end{array}\right\}=1$. Therefore, by the inductive supposition, we have

$$
\left\{\begin{array}{c}
n+1 \\
2^{r+1}-1
\end{array}\right\}=\left(\begin{array}{c}
n+1 \\
r+1
\end{array}\right)-\left(\begin{array}{l}
n \\
r
\end{array}\right)=\left(\begin{array}{c}
n \\
r+1
\end{array}\right)
$$

Another formula of the same type is

$$
\left(\begin{array}{l}
n \\
r
\end{array}\right)=\left\{\begin{array}{c}
n \\
2^{r-1}
\end{array}\right\}+1
$$

which follows from (3.1), if we set $m=r, k=l=0$ and replace $n$ by $n-r$. As a corollary, from (3.13)-(3.14) we have the equality

$$
\left\{\begin{array}{c}
n+1 \\
2^{r}-1
\end{array}\right\}=\left\{\begin{array}{c}
n \\
2^{r-1}
\end{array}\right\}+1
$$

We conclude this section by calculating some partial sums of the up-down coefficients. By (3.12)-(3.14), we have

$$
\begin{aligned}
\sum_{1 \leq r \leq n}\left\{\begin{array}{c}
n \\
2^{r-1}-1
\end{array}\right\} & =2^{n-1} ; \\
\sum_{1 \leq r \leq n}\left\{\begin{array}{c}
n \\
2^{r-1}
\end{array}\right\} & =2^{n}-n-1 ; \\
\sum_{1 \leq r \leq n}\left\{\begin{array}{c}
n \\
2^{r-1}+1
\end{array}\right\} & =2^{n-1}(n-2)-n^{2}+n+1 .
\end{aligned}
$$

It follows from (3.16) that the number of $\pi \in \mathbf{S}_{n}$ with exactly one change of sign in their signature is

$$
2\left(\sum_{1 \leq r \leq n}\left\{\begin{array}{c}
n \\
2^{r-1}-1
\end{array}\right\}-2\right)=2 \sum_{1 \leq r \leq n-2}\left\{\begin{array}{c}
n \\
2^{0}+2^{1}+\cdots+2^{r-1}
\end{array}\right\}=2^{n}-4, \quad n \geq 3 .
$$

Theorem 2. For $1 \leq r \leq n-1$ we have

$$
\sum_{k=0}^{2^{r}-1}\left\{\begin{array}{l}
n \\
k
\end{array}\right\}=n(n-1) \ldots(n-r+1)
$$


Proof. Sum (3.16) enumerates the permutations with $n-r-1$ fixed down points: 1, 2, $\ldots, n-r-1$. Let us form an arbitrary permutation $\pi$ of such a kind. We start with position $n-r+1$. We can choose the value of $\pi_{n-r+1}$ by $n$ ways, $\pi_{n-r+2}$ by $n-1$ ways, $\ldots, \pi_{n}$ by $n-(r-1)$ ways. After that $\pi_{1}>\pi_{2}>\cdots>\pi_{n-r}$ are defined uniquely. Thus, we obtain the required formula.

Now, as in the proof of properties (d)-(e), we have

$$
\sum_{\substack{0 \leq k \leq 2^{r}-1, k \text { even }}}\left\{\begin{array}{l}
n \\
k
\end{array}\right\}=\sum_{\substack{0 \leq k \leq 2^{r}-1, k \text { odd }}}\left\{\begin{array}{l}
n \\
k
\end{array}\right\}=\frac{1}{2} n(n-1) \cdots(n-r+1), \quad 2 \leq r \leq n-1 .
$$

This generalizes property (e) which corresponds to the case $r=n-1$.

Furthermore, note that, using (3.13), for a real $x$ we have

$$
\sum_{0 \leq k \leq n}\left\{\begin{array}{c}
n+1 \\
2^{k}-1
\end{array}\right\} x^{k}=\sum_{0 \leq k \leq n}\left(\begin{array}{l}
n \\
k
\end{array}\right) x^{k}=(x+1)^{n} .
$$

Thus

$$
\sum_{0 \leq k, l \leq n}\left\{\begin{array}{c}
n+1 \\
2^{k}-1
\end{array}\right\}\left\{\begin{array}{c}
n+1 \\
2^{l}-1
\end{array}\right\} x^{k+l}=(x+1)^{2 n}=\sum_{0 \leq m \leq 2 n}\left\{\begin{array}{l}
2 n+1 \\
2^{m}-1
\end{array}\right\} x^{m}
$$

Comparing coefficients of $x^{n}$, we find

$$
\sum_{0 \leq k \leq n}\left\{\begin{array}{c}
n+1 \\
2^{k}-1
\end{array}\right\}\left\{\begin{array}{c}
n+1 \\
2^{n-k}-1
\end{array}\right\}=\left\{\begin{array}{c}
2 n+1 \\
2^{n}-1
\end{array}\right\} .
$$

According to property (b) and (3.10),

$$
\left\{\begin{array}{c}
n+1 \\
2^{n-k}-1
\end{array}\right\}=\left\{\begin{array}{c}
n+1 \\
2^{n-1}-2^{n-k}
\end{array}\right\}=\left\{\begin{array}{c}
n+1 \\
2^{k}-1
\end{array}\right\} .
$$

This implies the formula

$$
\sum_{0 \leq k \leq n}\left\{\begin{array}{c}
n+1 \\
2^{k}-1
\end{array}\right\}^{2}=\left\{\begin{array}{c}
2 n+1 \\
2^{n}-1
\end{array}\right\}
$$

\section{Inequalities for up-down coefficients}

Now we find a series of inequalities for up-down coefficients.

Theorem 3. If $n \geq 1, \quad 0 \leq k<2^{n-1}, \quad m \geq 1, \quad 0 \leq l<2^{m-1}$, then we have

$$
\begin{aligned}
\left\{\begin{array}{c}
n+m+1 \\
2^{m} \cdot 2 k+l
\end{array}\right\} & <\left(\begin{array}{c}
n+m+1 \\
2^{m}(2 k+1)-l-1
\end{array}\right) ; \\
\left\{\begin{array}{c}
n+2 \\
4 k
\end{array}\right\} & <\left\{\begin{array}{c}
n+2 \\
4 k+1
\end{array}\right\} ; \\
\left\{\begin{array}{c}
n+2 \\
k
\end{array}\right\} & <\left\{\begin{array}{c}
n+2 \\
2^{n}-k-1
\end{array}\right\}=\left\{\begin{array}{c}
n+2 \\
2^{n}+k
\end{array}\right\} .
\end{aligned}
$$


Proof. There exist two permutations with signature $Q \in\{ \pm 1\}^{n-1}$, index $k$ and $P \in$ $\{ \pm 1\}^{m-1}$, index $l$. By Niven's theorem 5 [11], we have

$$
[Q,-1,-1, P]<[Q,-1,1,-P] .
$$

Since $-P$ has index $2^{m-1}-1-l$, we get (4.1). The other inequalities are the special cases $P=\varnothing$ and $Q=\varnothing$.

Remark 2. One cannot delete the restrictions $0 \leq k<2^{n-1}, 0 \leq l<2^{m-1}$ : e.g., $\left\{\begin{array}{c}4 \\ 20\end{array}\right\}=-3>-5=\left\{\begin{array}{c}4 \\ 21\end{array}\right\}$; but see (4.2).

Corollary 2. If $0 \leq k<2^{n-1}, \quad 0 \leq l<2^{m-1}$, then we have

$$
\begin{aligned}
& \left\{\begin{array}{c}
n+m+1 \\
l
\end{array}\right\}<\left\{\begin{array}{c}
n+m+1 \\
2^{m}-l-1
\end{array}\right\} \\
& \left\{\begin{array}{c}
n+m+1 \\
2^{m+n}-2^{m+1}+l
\end{array}\right\}<\left\{\begin{array}{c}
n+m+1 \\
2^{m+n}-2^{m}-l-1
\end{array}\right\} \text {; } \\
& \left\{\begin{array}{c}
n+m+1 \\
2^{m+1} k
\end{array}\right\}<\left\{\begin{array}{c}
n+m+1 \\
2^{m+1} k+2^{m}-1
\end{array}\right\} ; \\
& \left\{\begin{array}{c}
n+m+1 \\
2^{m+1} k+2^{m-1}-1
\end{array}\right\}<\left\{\begin{array}{c}
n+m+1 \\
2^{m+1} k+2^{m-1}
\end{array}\right\} \text {; } \\
& \left\{\begin{array}{c}
2 n+1 \\
2^{n+1} k+l
\end{array}\right\}<\left\{\begin{array}{c}
2 n+1 \\
2^{n+1} k+2^{n}-l-1
\end{array}\right\} ; \\
& \left\{\begin{array}{c}
n+1 \\
4 k+3
\end{array}\right\}<\left\{\begin{array}{c}
n+1 \\
4 k+2
\end{array}\right\} \\
& \left\{\begin{array}{c}
n+1 \\
3 \cdot 2^{n-1}+k
\end{array}\right\}<\left\{\begin{array}{c}
n+1 \\
3 \cdot 2^{n-1}-k-1
\end{array}\right\} \text {. }
\end{aligned}
$$

Remark 3. Note that, by direct computation, one can prove many special inequalities, for example,

$$
\begin{gathered}
\left\{\begin{array}{l}
n \\
4
\end{array}\right\}<\left\{\begin{array}{l}
n \\
6
\end{array}\right\}, \quad \text { if } n \geq 5, \\
\left\{\begin{array}{c}
n \\
12
\end{array}\right\}>\left\{\begin{array}{c}
n \\
16
\end{array}\right\}, \quad \text { if } n \geq 5 .
\end{gathered}
$$

\section{Maximal up-down coefficient}

Consider the sets of permutations of $n$ elements with a fixed signature. Which of these sets has the maximal cardinality? De Bruijn [5] mentions that Niven had a proof that it is the set of the alternating permutations. Unfortunately, Niven never published this statement. In this section we give a short proof of this remarkable fact. Note that the alternating permutation $\pi=\left(\pi_{1}, \pi_{2}, \ldots, \pi_{n}\right) \in \mathbf{S}_{n}$ with $\pi_{1}<\pi_{2}$ has index

$$
k_{n-1}:= \begin{cases}2^{n-2}+2^{n-4}+\cdots+2=\frac{1}{3}\left(2^{n}-2\right), & \text { if } n \text { is odd, } \\ 2^{n-2}+2^{n-4}+\cdots+1=\frac{1}{3}\left(2^{n}-1\right), & \text { if } n \text { is even. }\end{cases}
$$


Theorem 4. For $n \geq 1, \quad 0 \leq k<2^{n-1}$, we have

$$
\left\{\begin{array}{l}
n \\
k
\end{array}\right\} \leq\left\{\begin{array}{c}
n \\
k_{n-1}
\end{array}\right\}
$$

The equality holds if and only if $k=k_{n-1}$ or $k=2^{n-1}-1-k_{n-1}$.

Proof. Let $\pi=\left(\pi_{1}, \pi_{2}, \ldots, \pi_{n}\right) \in \mathbf{S}_{n}$ with index $k$. We assume $\pi_{1}<\pi_{2}$, hence sign $\pi=\left(1, q_{2}, q_{3}, \ldots, q_{n-1}\right)$. Now we use Niven's inequalities (4.4) and

$$
[Q, 1,1, P]<[Q, 1,-1,-P]
$$

If $q_{2} \neq-1$, then we replace it by -1 and, by (5.2) with $Q=\varnothing$, we get

$$
\left[1,1, q_{3}, \ldots, q_{n-1}\right]<\left[1,-1,-q_{3}, \ldots,-q_{n-1}\right] \text {. }
$$

Hence we can assume $q_{2}=-1$. If $-q_{3} \neq 1$, then we replace $-q_{3}$ by 1 and, by (4.4), we get

$$
\left[1,-1,-1,-q_{4}, \ldots,-q_{n-1}\right]<\left[1,-1,1, q_{4}, \ldots, q_{n-1}\right] .
$$

So we can continue until we obtain an alternating permutation. If the initial permutation is not alternating, then we need at least one step and get " $<$ ". Thus we have the equality only in the case $k=k_{n-1}$. Note that, in the second case, when we suppose that $\pi_{1}>\pi_{2}$, we obtain an alternating permutation with $k=2^{n-1}-1-k_{n-1}$.

Note that the signature of an alternating permutation has the maximal number of changes of signs. One may expect that $\left\{\begin{array}{l}n \\ k\end{array}\right\}$ grows together with the number of changes. But this is wrong. Indeed, consider, e.g., permutations $(4,3,2,1,5,6,7,8) \in \mathbf{S}_{8}$ with index $2^{3}+$ $2^{2}+2+1=15$ and $(1,8,2,3,4,5,6,7) \in \mathbf{S}_{8}$ with index $2^{6}+2^{4}+2^{3}+2^{2}+2+1=95$. The first permutation has only one change, while the latter has two. However, $\left\{\begin{array}{c}8 \\ 15\end{array}\right\}=35>$ $27=\left\{\begin{array}{c}8 \\ 95\end{array}\right\}$. If, however, we consider $2^{3}+2=10$, then we find $\left\{\begin{array}{c}8 \\ 15\end{array}\right\}=35<323=\left\{\begin{array}{c}8 \\ 10\end{array}\right\}$. In connection with these examples, we prove the following more general result.

Theorem 5. Let $n \geq 1, \quad 0 \leq k<2^{n-1}, \quad 1 \leq m<n$. Then

$$
\left\{\begin{array}{l}
n \\
k
\end{array}\right\} \leq \max _{i \in\{m-1, m\}}\left\{\begin{array}{c}
n \\
\left\lfloor\frac{k}{2^{m}}\right\rfloor 2^{m}+k_{i}
\end{array}\right\} \text {. }
$$

Proof. The case $m=n-1$ is contained in Theorem 4. Let $m<n-1$ and

$$
k=\sum_{0 \leq i \leq n-2} a_{i} 2^{i}=\left\lfloor\frac{k}{2^{m}}\right\rfloor 2^{m}+a_{m-1} 2^{m-1}+\cdots+a_{0} .
$$

If $a_{m}=0$, we have

$$
\left\{\begin{array}{l}
n \\
k
\end{array}\right\} \leq\left\{\begin{array}{c}
n \\
\left\lfloor\frac{k}{2^{m}}\right\rfloor 2^{m}+(1010 \ldots)_{2}
\end{array}\right\}=\left\{\begin{array}{c}
n \\
\left\lfloor\frac{k}{2^{m}}\right\rfloor 2^{m}+k_{m}
\end{array}\right\} .
$$


If $a_{m}=1$, then

$$
\left\{\begin{array}{l}
n \\
k
\end{array}\right\} \leq\left\{\begin{array}{c}
n \\
\left\lfloor\frac{k}{2^{m}}\right\rfloor 2^{m}+(0101 \ldots)_{2}
\end{array}\right\}=\left\{\begin{array}{c}
n \\
\left\lfloor\frac{k}{2^{m}}\right\rfloor 2^{m}+k_{m-1}
\end{array}\right\} .
$$

This proves the theorem.

Example. $n=8, k=15, m=3$ : in the proof $a_{3}=1$, hence $\left\{\begin{array}{c}8 \\ 15\end{array}\right\}<\left\{\begin{array}{c}8 \\ 10\end{array}\right\}$.

If in Theorem 5 we replace $n$ by $n+m$ and $k$ by $2^{m} k+l$, then we get

Corollary 3. Let $n, m \geq 1, \quad 0 \leq k<2^{n-1}, \quad 0 \leq l<2^{m-1}$. Then we have

$$
\left\{\begin{array}{c}
n+m \\
2^{m} k+l
\end{array}\right\} \leq \max _{i \in\{m-1, m\}}\left\{\begin{array}{c}
n+m \\
2^{m} k+k_{i}
\end{array}\right\} \text {. }
$$

\section{Up-down coefficients and numbers of Euler and Bernoulli}

In 1966, Entringer [7] for the first time indicated a simple connection of the numbers of alternating permutations with the Bernoulli and Euler numbers. We give a short proof of this result in our terminology.

Theorem 6. For every $n \in \mathbb{N}$, we have

$$
\left\{\begin{array}{c}
2 n \\
k_{2 n-1}
\end{array}\right\}=\left|E_{2 n}\right|, \quad\left\{\begin{array}{c}
2 n-1 \\
k_{2 n-2}
\end{array}\right\}=\left|b_{2 n}\right| .
$$

Proof. The Euler numbers $E_{2 n}$ can be defined by their generating function

$$
\sum_{n \geq 0}(-1)^{n} E_{2 n} \frac{x^{2 n}}{(2 n) !}=\frac{1}{\cos x}, \quad|x|<\frac{\pi}{2}
$$

and the Bernoulli numbers $b_{2 n}$ by

$$
\sum_{n \geq 1}(-1)^{n} b_{2 n} \frac{x^{2 n-1}}{(2 n-1) !}=\tan x .
$$

Using André's result [2], for the generating function of the up-down coefficients $\left\{\begin{array}{c}n \\ k_{n-1}\end{array}\right\}$ we have

$$
f(x):=1+\sum_{n \geq 1}\left\{\begin{array}{c}
n \\
k_{n-1}
\end{array}\right\} \frac{x^{n}}{n !}=\tan x+\frac{1}{\cos x}, \quad|x|<\frac{\pi}{2} .
$$

Separating symmetric and antisymmetric parts, we find for $|x|<\frac{\pi}{2}$

$$
1+\sum_{n \geq 1}\left\{\begin{array}{c}
2 n \\
k_{2 n-1}
\end{array}\right\} \frac{x^{2 n}}{(2 n) !}=\frac{1}{\cos x} \quad \text { and } \quad \sum_{n \geq 1}\left\{\begin{array}{c}
2 n-1 \\
k_{2 n-2}
\end{array}\right\} \frac{x^{2 n-1}}{(2 n-1) !}=\tan x .
$$

By comparing the coefficients with (6.1)-(6.2), we get the desired results

$$
\left\{\begin{array}{c}
2 n \\
k_{2 n-1}
\end{array}\right\}=(-1)^{n} E_{2 n}=\left|E_{2 n}\right|, \quad\left\{\begin{array}{c}
2 n-1 \\
k_{2 n-2}
\end{array}\right\}=(-1)^{n} b_{2 n}=\left|b_{2 n}\right| .
$$


Now we are able to get a non-trivial upper estimate for the up-down coefficients.

Theorem 7. Uniformly on $k$, we have

$$
\left\{\begin{array}{l}
n \\
k
\end{array}\right\}<\frac{\left(2^{n+2}-2\right) n !}{\left(1-2^{-n}\right) \pi^{n+1}}, \quad n=1,2, \ldots .
$$

Proof. As is well known,

$$
\tan x=\sum_{n \geq 1} 2^{2 n}\left(2^{2 n}-1\right)\left|B_{2 n}\right| \frac{x^{2 n-1}}{(2 n) !}
$$

where $B_{2 n}$ are Bernoulli numbers which, in contrast to $b_{2 n}$, are defined by the generating function

$$
\frac{x}{e^{x}-1}=1+\sum_{n \geq 1} B_{2 n} \frac{x^{2 n}}{(2 n) !} .
$$

Comparing with (6.2), we conclude that

$$
\left|b_{2 n}\right|=\frac{2^{2 n}\left(2^{2 n}-1\right)}{2 n}\left|B_{2 n}\right|, \quad n=1,2, \ldots
$$

The following inequalities for the Bernoulli and Euler numbers are known ([1], cf. formulas 23.1.15):

$$
\begin{gathered}
\left|E_{n}\right|<\frac{2^{n+2} n !}{\pi^{n+1}}, \quad n=2,4, \ldots, \\
\left|B_{n+1}\right|<\left(\frac{1}{1-2^{-n}}\right) \frac{2(n+1) !}{(2 \pi)^{n+1}}, \quad n=1,3, \ldots .
\end{gathered}
$$

Multiplying the latter by $\frac{2^{n+1}\left(2^{n+1}-1\right)}{n+1}$, from (6.6) we find

$$
\left|b_{n+1}\right|<\left(\frac{1}{1-2^{-n}}\right) \frac{\left(2^{n+2}-2\right) n !}{\pi^{n+1}}, n=1,3, \ldots .
$$

Since

$$
2^{n+2}<\frac{2^{n+2}-2}{1-2^{-n}}
$$

by Theorems 4, 6 and formulas (6.6) and (6.9), we have

$$
\left\{\begin{array}{c}
n \\
k_{n-1}
\end{array}\right\}<\frac{\left(2^{n+2}-2\right) n !}{\left(1-2^{-n}\right) \pi^{n+1}}, \quad n=1,2, \ldots
$$

and the required estimate follows from Theorem 4. 


\section{Several recurrent relations}

There are many linear relations between the Bernoulli and Euler numbers ([9]). According to Theorem 6, every one leads to a recursion for the maximal up-down coefficients. For example, if in $b_{2 n}=-1-\sum_{1 \leq r<n}\left(\begin{array}{c}2 n-1 \\ 2 r\end{array}\right) E_{2 r}$ we replace $b_{2 n}=(-1)^{n}\left\{\begin{array}{c}2 n-1 \\ k_{2 n-2}\end{array}\right\}$ and $E_{2 r}=$ $(-1)^{r}\left\{\begin{array}{c}2 r \\ k_{2 r-1}\end{array}\right\}$, we get for $n \geq 2$

$$
\left\{\begin{array}{c}
2 n-1 \\
k_{2 n-1}
\end{array}\right\}=(-1)^{n-1}+\sum_{1 \leq r<n}(-1)^{n-r+1}\left(\begin{array}{c}
2 n-1 \\
2 r
\end{array}\right)\left\{\begin{array}{c}
2 r \\
k_{2 r-1}
\end{array}\right\} .
$$

In a similar way, using $E_{2 n}=1+\sum_{1 \leq r \leq n}\left(\begin{array}{c}2 n \\ 2 r-1\end{array}\right) b_{2 r}$, we have $\left(k_{0}=0\right)$

$$
\left\{\begin{array}{c}
2 n \\
k_{2 n-1}
\end{array}\right\}=(-1)^{n}+\sum_{1 \leq r \leq n}(-1)^{n-r}\left(\begin{array}{c}
2 n \\
2 r-1
\end{array}\right)\left\{\begin{array}{c}
2 r-1 \\
k_{2 r-2}
\end{array}\right\}, n \geq 1 .
$$

The next two recursions allow to calculate $\left\{\begin{array}{c}2 n \\ k_{2 n-1}\end{array}\right\}$ and $\left\{\begin{array}{c}2 n-1 \\ k_{2 n-1}\end{array}\right\}$ independently. We have

$$
\begin{aligned}
\left\{\begin{array}{c}
2 n \\
k_{2 n-1}
\end{array}\right\} & =(-1)^{n-1}+\sum_{1 \leq r<n}(-1)^{n-r+1}\left(\begin{array}{c}
2 n \\
2 r
\end{array}\right)\left\{\begin{array}{c}
2 r \\
k_{2 r-1}
\end{array}\right\} ; \\
\left\{\begin{array}{c}
2 n-1 \\
k_{2 n-2}
\end{array}\right\} & =(-1)^{n-1}+\sum_{1 \leq r<n}(-1)^{n-r+1}\left(\begin{array}{c}
2 n-1 \\
2 r-1
\end{array}\right)\left\{\begin{array}{c}
2 r-1 \\
k_{2 r-2}
\end{array}\right\} .
\end{aligned}
$$

They come from $E_{2 n}=-\sum_{0 \leq r<n}\left(\begin{array}{c}2 n \\ 2 r\end{array}\right) E_{2 r}$ and $b_{2 n}=-1-\sum_{1 \leq r<n}\left(\begin{array}{c}2 n-1 \\ 2 r-1\end{array}\right) b_{2 r}$ correspondingly.

\section{Acknowledgment}

The authors are grateful to P.J.C. Moses (UK) for improvements in the text. They also thank a referee for several useful remarks.

\section{References}

[1] M. Abramowitz and I.A. Stegun (Eds.), Bernoulli and Euler Polynomials and the Euler-Maclaurin Formula in Handbook of Mathematical Functions with Formulas, Graphs, and Mathematical Tables, 9th printing, New York: Dover, pp. 804-806, 1972.

[2] D. André, Sur les permutations alternées, J. de Math. (3), 7 (1881),167-184.

[3] V. Arnold, Bernoulli-Euler updown numbers associated with function singularities, their combinatorics and arithmetic, Duke Math. J., 63(2) (1991), 537-555.

[4] F.C.S. Brown, T.M.A. Fink, K. Willbrand, On arithmetic and asymptotic properties of up-down numbers, Discrete Math., 307 (2007), 1722-1736.

[5] N.G. de Bruijn, Permutations with given ups and downs, Nieuw Arch., 18 (1970), 61-65.

[6] L. Carlitz, Permutations with prescribed pattern, II, Math. Nachr. 83 (1978), 101-126.

[7] R.C. Entringer, A combinatorial interpretation of the Euler and Bernoulli numbers, Nieuw Arch. Wisk. (3), 14 (1966), 241-246. 
[8] H.O. Foulkes, Enumeration of permutations with prescribed up-down and inversion sequences, Discrete Math., 15 (1976), 235-252.

[9] A. Jeffrey and D. Zwillinger (eds.), Gradshteyn and Ryzhik's table of integrals, series and products, Seventh edition (2007).

[10] C.L. Mallows and L.A. Shepp, Enumerating pairs of permutations with the same up-down form, Discrete Math. 54 (1985), 301-311.

[11] I. Niven, A combinatorial problem of finite sequences, Nieuw Arch. Wisk (3) 16 (1968), 116-123.

[12] B. Shapiro, M. Shapiro, A. Vainshtein, Periodic de Bruijn triangles: exact and asymptotic results, Discrete Math., 298(1-3) (2005), 321-333.

[13] V. Shevelev, Number of permutations with prescribed up-down structure as a function of two variables, INTEGERS 12(2012), article \#A1.

[14] R. Stanley, Alternating permutations and symmetric functions, J. Combin. Theory Series A, 114 (2007), 436-460.

[15] G. Szpiro, The number of permutations with a given signature and the expectations of their elements, Discrete Math., 226 (2001), 423-430.

[16] G. Viennot, Permutations ayant une forme donnée. Discrete Math., 26 (1979), 279-284.

Vladimir Shevelev

Department of Mathematics

Box 142

Ben Gurion University of the Negev

POB 653

Beer-Sheva 84105, Israel

e-mail: shevelev@bgu.ac.il

Juergen Spilker

Am Schloßpark 10

D-79252 Stegen, Deutschland

e-mail: juergen.spilker@t-online.de 